\title{
Comparison of Performance and Computational Complexity of Nonlinear Active Noise Control Algorithms
}

\author{
Mouayad A. Sahib and Raja Kamil \\ Department of Electrical and Electronic Engineering, University Putra Malaysia, 43400 Serdang, Selangor, Malaysia \\ Correspondence should be addressed to Raja Kamil, kamil@eng.upm.edu.my \\ Received 21 May 2011; Accepted 15 June 2011 \\ Academic Editors: F. Liu and J.-L. Marcelin
}

Copyright (๑) 2011 M. A. Sahib and R. Kamil. This is an open access article distributed under the Creative Commons Attribution License, which permits unrestricted use, distribution, and reproduction in any medium, provided the original work is properly cited.

\begin{abstract}
Research on nonlinear active noise control (NANC) revolves around the investigation of the sources of nonlinearity as well as the performance and computational load of the nonlinear algorithms. The nonlinear sources could originate from the noise process, primary and secondary propagation paths, and actuators consisting of loudspeaker, microphone or amplifier. Several NANCs including Volterra filtered-x least mean square (VFXLMS), bilinear filtered-x least mean square (BFXLMS), and filtered$s$ least mean square (FSLMS) have been utilized to overcome these nonlinearities effects. However, the relative performance and computational complexities of these algorithm in comparison to FXLMS algorithm have not been carefully studied. In this paper, systematic comparisons of the FXLMS against the nonlinear algorithms are evaluated in overcoming various nonlinearity sources. The evaluation of the algorithms performance is standardized in terms of the normalized mean square error while the computational complexity is calculated based on the number of multiplications and additions in a single iteration. Computer simulations show that the performance of the FXLMS is more than $80 \%$ of the most effective nonlinear algorithm for each type of nonlinearity sources at the fraction of computational load. The simulation results also suggest that it is more advantageous to use FXLMS for practical implementation of NANC.
\end{abstract}

\section{Introduction}

The increase in environmental noise and the need for a low-cost noise control system promote the growth of active noise control (ANC) applications. Transportation and road noise from motor vehicles, aircraft, and rail are major contributors to environmental noise [1]. An example of transportation noise is the ambient noise produced by hybrid electrical vehicles, which originates from the alternator and cooling fans of the engine and battery packs [2]. Large wind turbines with large blade propellers create a low-frequency drumming that penetrates surrounding residential areas [3]. The conventional method to reduce the amount of noise involves the application of passive absorbers based on the concept of energy loss [4]. However, for low-frequency noise, this method is not effective [5], and ANC is resorted to instead. The principle of ANC is to produce a secondary destructive antinoise signal equal in magnitude but opposite in phase electronically to cancel the primary unwanted noise $[6,7]$.
Linear ANC techniques are limited in that the system exhibits performance degradation when dealing with nonlinearities $[1,8-10]$. Nonlinearity is a concern in ANC application where low-cost devices such as amplifiers, sensors, and actuators exhibit nonlinear distortion. Therefore, using low-cost sensors and actuators in an ANC system along with an efficient NANC algorithm can reduce the cost of the control system while ensuring good cancellation level. In the literature concerning NANC systems, two main models are commonly used to represent nonlinearities, namely, the truncated Volterra series $[8,9,11,12]$ and functional link neural network (FLNN) $[5,7,11-16]$. A study has suggested that the output error Bilinear filter is an efficient alternative to Volterra filter in reducing the saturation effect of the sensor and actuator [17]. In the FLNN models, different functional expansions have been implemented with the trigonometric expansion being the most common $[7,12,13]$. Other expansions include piecewise [15], Chebyschev [11, 12, 14], truncated Taylor series for trigonometric functions [14], Legendre [14], and 
Hammerstein [14]. In addition to the FLNN structure, two other adaptive feedback FLNN filters have been proposed to reduce the effects of nonlinearities in the primary path and noise process with lower computational loads. They are the feedback FLNN (FFLNN) and reduced FFLNN (RFFLNN) [13]. Other models in the literature include fuzzy neural networks (FNN) [18], and nonlinear autoregressive moving average with exogenous input (NARMAX) models [6].

The nonlinear models used in NANC can be updated adaptively using various algorithms. In this paper, the performance of the nonlinear VFXLMS, BFXLMS and FSLMS algorithms are compared with the linear FXLMS, algorithms in terms of noise reduction and computational load. The algorithm with the best performance for each type of nonlinearity is identified. The paper is organized as follows: Section 2 describes the sources of nonlinearity in the ANC system. In Section 3 the feedforward ANC structure is presented. Section 4 describes the three NANC algorithms. The simulation results of the performance of the algorithms are presented in Section 5. The computational complexity of the algorithms is presented in Section 6 followed by the conclusion in Section 7.

\section{Sources of Nonlinearities}

The nonlinearity sources in active noise control can be generally classified into three types. The first type is due to system actuators such as the loudspeaker, microphones, preamplifiers, analogue to digital converters (ADC), and digital to analogue converters (DAC). The second type is due to the noise process when the dynamic behaviour of the noise generation is nonlinear. The third type is due to the acoustic propagation paths, where the acoustic signals propagate under the presence of nonlinearities, such as high pressure, temperature variations, or nonhomogeneous media [19]. The following subsections describe the three nonlinearity types and the relevant mathematical models commonly used to represent them.

2.1. Sensors, Actuators, and Amplifiers. In many practical ANC applications, the actuator that generates the antinoise signal is a loudspeaker, and the sensor that detects the error signal is a microphone. It is possible that the primary noise amplitude level is so high that the error and reference sensors are saturated due to their low-power characteristics. The saturation and clipping effects due to over driving the loudspeakers can produce extra odd harmonics, thus, affecting the convergence speed of the NANC controller [20]. Aging and corrosion of electronic components due to temperature variations and humidity in ADCs and DACs circuits are additional nonlinear sources [20].

In loudspeakers, the nonlinearity in the form of limited dynamic range causes electrical distortion when output amplitude level is high. The two most important forms of distortion in the loudspeakers are (i) harmonic distortion and (ii) intermodulation distortion [21]. Harmonic distortion is characterized by the presence of harmonics in the output signal not present in the original (excitation) signal. On the other hand, intermodulation distortion arises whenever two signals having different frequencies pass simultaneously through a nonlinear system. As a result, linear combinations of the fundamental frequencies and all harmonics present in the input signals may appear as an overall nonlinearity at the loudspeaker output.

In order to deliver sufficient power to drive the loudspeaker, power amplifier is used to amplify the control signal. The amplifier should ideally provide nominally flat response between $20 \mathrm{~Hz}$ and $20 \mathrm{kHz}$ and generate a minimum harmonic distortion. However, nonlinear harmonic distortion which consists mainly of cubic terms having 5 to 10 percent amplitude of the total output amplitude can occur, especially when dealing with small loudspeakers that operate at high volumes [22].

The nonlinearities of the power amplifier, loudspeaker, microphone, and preamplifier can be represented as a lumped model system using second-order Volterra series [23]. The Volterra expansion is a general method used to model nonlinear systems including saturation-type nonlinearities observed in power amplifiers and loudspeakers. The nonlinear characteristic of an amplifier loudspeaker can be represented by a NARMAX model [21]. Typically, the saturation characteristic of a loudspeaker is modelled by the sigmoid hyperbolic tangent function. In previous study, the nonlinear effect of the power amplifier and loudspeaker has been modelled as a nonlinear filter without memory [1] which is defined as

$$
r(n)=0.66 \tanh \{1.5 y(n)\}
$$

However, the representation in (1) cannot be used to approximate all types of nonlinear characteristics of the amplifier-loudspeaker system due to its fixed amplitude terms.

A raised cosine function has also been used to model the nonlinear characteristics of the amplifier-loudspeaker system [24]. In the function, two parameters can be adjusted to influence the shape of the raised cosine function. The adjustable raised cosine function improves the approximation of the nonlinear characteristics compared to the sigmoid hyperbolic tangent function given by (1).

2.2. Reference Noise. Recently, chaotic signal processing has received much interest. Some signals, like radar sea clutter, speech, and indoor multipath effects, are better represented by deterministic chaotic rather than stochastic process. Understanding the chaotic process is useful in applications such as radar surveillance, secure communication, and narrowband interference cancellation in chaotic spreadspectrum system $[25,26]$. The main characteristic of a deterministically chaotic system is the sensitive dependence on initial conditions. Small differences in the initial input conditions can grow into large changes in the output value. Moreover, it is very difficult to predict the next system state, even if an accurate measurement of the current state can be obtained [27].

In ANC system, the noise that is generated from a dynamic system may be nonlinear and deterministic chaotic rather than stochastic, white, or tonal noise processes 
$[1,5,7,8,12,14,16-18]$. Research has shown that the noise measured from a ventilation fan exhibit chaotic behaviour [28]. Three kinds of chaotic noises in the form of Logistic, Lorenz, and Duffing noise filters have been applied to test the capability of a proposed single channel nonlinear controller [29]. The results from this study show that if the input signal is non-Gaussian and predictable, the use of a combination of linear and nonlinear controller produces better results than a linear one exclusively.

Among these chaotic noises, the Logistic type is the simplest and the most useful test signal, and it can be described as a second-order white and predictable nonlinear process. The Logistic process can be generated using the following equation $[7,8,12,14,16-18]$ :

$$
x(n+1)=\lambda x(n)(1-x(n)) .
$$

The white noise signal $x(n)$ is typically generated by setting $\lambda$ and the initial condition $x(0)$ to be 4 and 0.9 , respectively $[1,5]$.

2.3. Propagation Paths. Primary and secondary propagation paths may exhibit nonlinear impulse responses. Nonlinear distortion between the reference sensor and the error sensor occurs in ducts where the noise propagates with high sound pressure [30]. Typically, a sinusoidal sound wave of $500 \mathrm{~Hz}$ propagating in a duct at a sound pressure level of $140 \mathrm{~dB}$ generates a harmonic distortion of about $1 \%$ after travelling a distance of $1 \mathrm{~m} \mathrm{[30].}$

The nonlinear noise source at the cancelling point can be represented by a third-order polynomial equation given as $[7,8,12,14]$

$$
d(n)=c(n-2)+0.08 c^{2}(n-2)-0.04 c^{3}(n-2) .
$$
tion

In $(3), c(n)$ is obtained by the following linear convolu-

$$
c(n)=x(n) * p(n),
$$

where $x(n)$ is the reference noise, and $p(n)$ represents the impulse response of the primary path expressed as

$$
p(n)=\delta(n-5)-0.3 \delta(n-6)+0.2 \delta(n-7) \text {. }
$$

In general, FXLMS structure and algorithm are used both in linear and nonlinear feedforward ANC systems. The reference signal must be filtered for correct adaptation. In the next section, the feedforward ANC with FXLMS algorithm is presented.

\section{Feedforward Active Noise Control}

The basic single channel ANC system can be represented using an adaptive filtering block diagram, as shown in Figure 1. One of the most popular adaptive algorithms applied in ANC is the filtered-x least mean square (FXLMS) algorithm [31]. This algorithm is a variant of the LMS algorithm. It is used when the reference signal needs to be

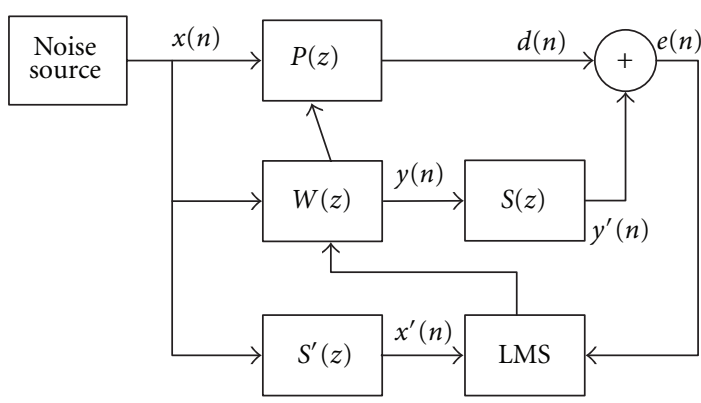

FIgURE 1: ANC System using FXLMS Algorithm.

filtered by the transfer function of the secondary path to ensure correct controller adaptation [32].

The FXLMS scheme is depicted in Figure 1, where $x(n)$, $x^{\prime}(n), y(n), y^{\prime}(n), d(n)$, and $e(n)$ are discrete time domain signals representing noise process, filtered noise, controller output, secondary path output, primary path output, and residual noise error signals, respectively. $P(z), W(z)$, $S^{\prime}(z)$, and $S(z)$ are the $z$-transforms for the transfer functions of the primary path, adaptive controller, estimated secondary path and secondary path systems, respectively. The objective of the adaptive controller is to generate an antinoise signal in order to minimize the residual error noise. The controller is typically an FIR filter with tuneable weights adjusted to minimize the error signal.

\section{NANC Algorithms}

The performance of linear ANC algorithm (FXLMS) degrades due to the existence of nonlinearity sources. Therefore, NANC algorithms are needed. Such algorithms are utilised in adapting nonlinear models such as truncated Volterra series, functional link neural networks, bilinear filters, and the NARMAX model. The model order represented by the number of weights is an important issue in hardware implementation. It affects both the computational load and the memory requirements of the control algorithm. Typically, in polynomial models such as the Volterra, bilinear, and NARMAX models, the model order is set to a large value causing over parameterization. However, only a few parameters are dominant to model the system. Therefore, several strategies have been employed to tackle the over parameterization problem like akaike information criterion (AIC) and bayesian information criterion (BIC) [6].

FLNN uses the functional expansion structure to replace the task performed by the hidden layers in traditional neural networks. This replacement has the advantage of making the structure for hardware easier to implement. Moreover, FLNN involves less computational complexity compared to the multilayer artificial neural network (MLANN).

This paper compares the performance and computational complexity of three NANC algorithms, which are commonly applied to update the nonlinear controllers. The algorithms considered are Volterra filtered-x least mean square (VFXLMS), bilinear filtered-x least mean square (BFXLMS), and filtered-s least mean square (FSLMS) which 
is based on the functional link neural network (FLNN) model. The description of the three NANC algorithms is given in the following subsections.

4.1. VFXLMS Based on Volterra Filters. The general Porder input-output relationship for a Volterra filter in a multichannel structure is given by $[8,9]$

$$
y(n)=\sum_{i=1}^{P} y_{i}(n)
$$

where $P$ is the order of the Volterra filter, and the term $y_{i}(n)$ can be further defined as

$$
\begin{aligned}
& y_{i}(n) \\
& =\sum_{m_{1}=0}^{M-1} \sum_{m_{2}=m_{1}}^{M-1} \ldots \sum_{m_{i}=m_{i-1}}^{M-1}\left\{h_{i}\left(m_{1}, m_{2}, \ldots, m_{i}\right) \prod_{k=1}^{i} x\left(n-m_{k}\right)\right\} .
\end{aligned}
$$

In (7), $M$ represents the maximum number of past input samples (memory length). The Volterra output $y(n)$ can be represented in vector form as

$$
y(n)=\mathbf{x}(n) \mathbf{H}^{T}(n)
$$

where

$$
\mathbf{x}(n)=\left[\bar{x}_{1}(n), \bar{x}_{2}(n), \ldots, \bar{x}_{P}(n)\right] .
$$

The terms in the bracket of (9) which represent the input signal to the filter can be written as

$$
\begin{aligned}
\bar{x}_{1}(n)= & x(n), x(n-1), \ldots, x(n-M)], \\
\bar{x}_{2}(n)= & {\left[x(n)^{2}, x(n) x(n-1), \ldots, x(n) x(n-M),\right.} \\
& x(n-1)^{2}, x(n-1) x(n-2), x(n-1) x(n-M), \\
& \left.x(n-2)^{2}, x(n-2) x(n-3), \ldots, x(n-M)^{2}\right], \\
\bar{x}_{P}(n)= & {\left[x(n)^{P}, x(n)^{P-1} x(n-1), \ldots, x(n)^{P-1} x(n-M),\right.} \\
& \left.x(n-1)^{P}, x(n-1)^{P-1} x(n-2), \ldots, x(n-M)^{P}\right],
\end{aligned}
$$

and the impulse response of the filters can be written as

$$
\mathbf{H}(n)=\left[h_{1}(n), h_{2}(n), \ldots, h_{P}(n)\right],
$$

where $h_{P}(n)$ is a vector that represents the coefficients of the $P$ th order Volterra kernel of the system. The Volterra filter can be updated using the VFXLMS algorithm as shown in Figure 2. The error signal between the secondary path output and the desired signal is given as

$$
e(n)=d(n)-s(n) * \sum_{i=1}^{P} y_{i}(n)=d(n)-s(n) * \mathbf{x}(n) \mathbf{H}^{T}(n),
$$

where the operation $(*)$ represents time-domain convolution. The coefficient vector will be adjusted accordingly to minimize the mean square error $\{e(n)\}^{2}$, which is equal to minimizing the residual noise power [9]

$$
\begin{aligned}
\mathbf{H}(n+1) & =\mathbf{H}(n)-\frac{\mu}{2} \frac{\partial e^{2}(n)}{\partial \mathbf{H}(n)}, \\
\frac{\partial e^{2}(n)}{\partial \mathbf{H}(n)} & =2 e(n) \frac{\partial e(n)}{\partial \mathbf{H}(n)} \\
& =2 e(n) \frac{\partial\left\{d(n)-s(n) * \mathbf{x}(n) \mathbf{H}^{T}(n)\right\}}{\partial \mathbf{H}(n)} \\
& =-2 e(n)\{s(n) * \mathbf{x}(n)\}=-2 e(n) \mathbf{x}_{\mathbf{f}}(n),
\end{aligned}
$$

where $\mathbf{x}_{\mathbf{f}}(n)$ is the $\mathbf{x}(n)$ signal filtered by the secondary path $s(n)$. Substituting (14) into (13) yields

$$
\mathbf{H}(n+1)=\mathbf{H}(n)+2 e(n) \mathbf{x}_{\mathbf{f}}(n) .
$$

The Volterra model typically requires a large number of parameters to estimate. In practice, the model order is initially chosen to be large which could cause overparameterization. Unfortunately, overparameterization will degrades parameter estimation accuracy and the robustness of the model. Moreover, overparameterization can be responsible for several unwanted dynamic effects [33]. On the positive side, Volterra model is linear in the parameters, such that conventional algorithms like least mean squares used for FIR filters adaptation be can still applied with minor modifications.

4.2. BFXLMS Based on Bilinear Filters. In comparison to FIR filter, IIR filter can model a linear system with fewer coefficients. These coefficients are associated with delayed input and output samples. The Volterra filters are viewed as the nonlinear generalisation of a linear FIR filter while bilinear filters are an extension of the linear IIR filter with additional coefficients associated with the input-output cross multiplied samples. Thus, bilinear filters can model nonlinear systems with fewer coefficients compared to Volterra filters. The general equation of the input-output relationship of bilinear filters is given as [17]

$$
\begin{aligned}
y(n)= & \sum_{i=0}^{M} A_{i}(n) x(n-i)+\sum_{j=0}^{L} B_{i}(n) y(n-j) \\
& +\sum_{i=0}^{M} \sum_{j=0}^{L} C_{i, j}(n) x(n-i) y(n-j),
\end{aligned}
$$

where $A_{i}(n), B_{i}(n)$, and $C_{i, j}(n)$ are the coefficients of the delayed input, delayed output, and delayed input-output cross-multiplied samples, respectively. $M$ and $L$ are the number of input and output delay taps, respectively. The block diagram of the BFXLMS for the feedforward ANC system is shown in Figure 3.

From Figure 3, the output of the bilinear filters is expressed as

$$
y(n)=x(n) * A(n)+y(n-1) * B(n)+x y(n) * C(n) .
$$




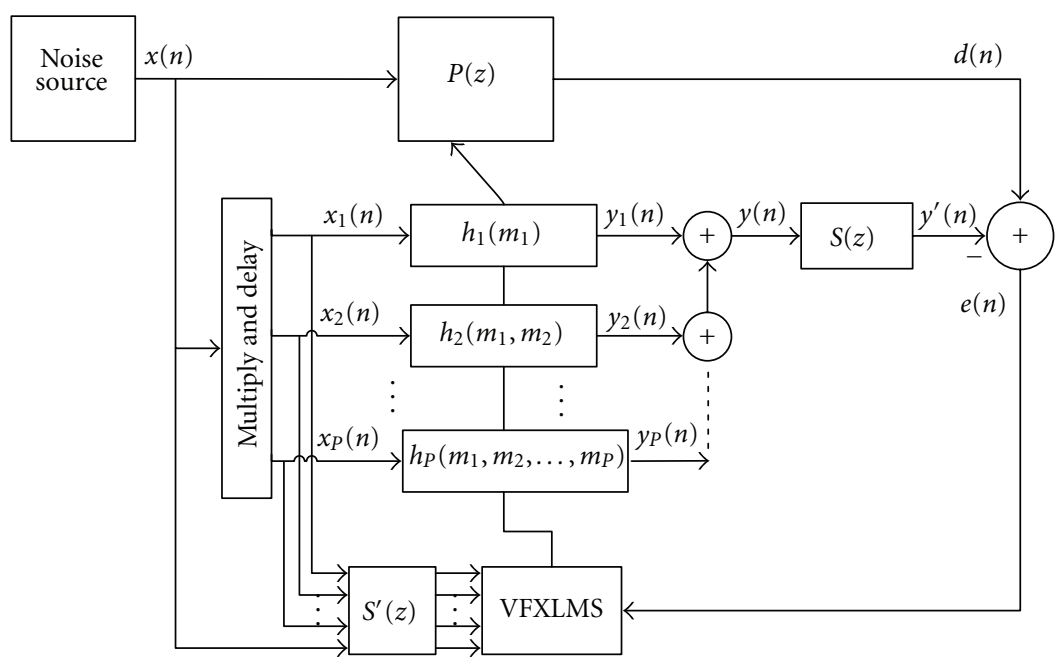

FIgURE 2: VFXLMS for the feedforward ANC system.

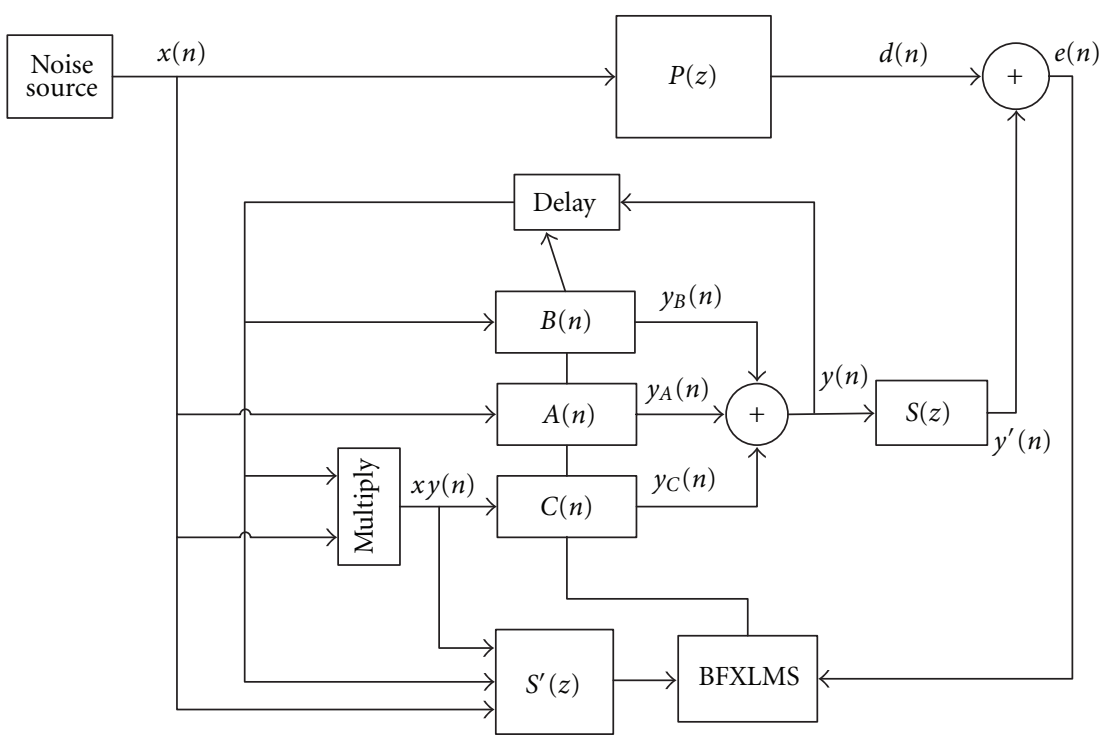

FIGURE 3: BFXLMS for the feedforward ANC system.

In BFXLMS, steepest descent algorithm is utilized in minimizing the squared error function. The weights are updated according to [17]

$$
\begin{gathered}
A(n)=A(n)+\mu_{A} e(n) x_{f}(n), \\
B(n)=B(n)+\mu_{B} e(n) y_{f}(n), \\
C(n)=C(n)+\mu_{C} e(n) x y_{f}(n),
\end{gathered}
$$

where $x_{f}(n), y_{f}(n)$, and $x y_{f}(n)$ are the filtered signals through the estimated secondary path block $S^{\prime}(z)$ of the input, output, and the input-output crossover multiplied delayed samples, respectively.

The disadvantages of modelling using the adaptive bilinear filters are the following.
(1) The adaptive bilinear filter may not converge to the global minimum because the error function has local minima.

(2) The adaptation process may be unstable.

These problems can be avoided if the algorithm is designed by choosing the step size carefully. In terms of computational complexity, BFXLMS requires less computation compared to VFXLMS for the same amount of noise reduction and same system conditions.

4.3. FSLMS Based on FLNN. In conventional MLANN, the activation functions are used to introduce nonlinearity into the network. In FLNN, the functional expansion carries out the task performed by the activation functions. As a result of this fact, FLNN has the key advantages of involving less computational complexity and a simpler structure 


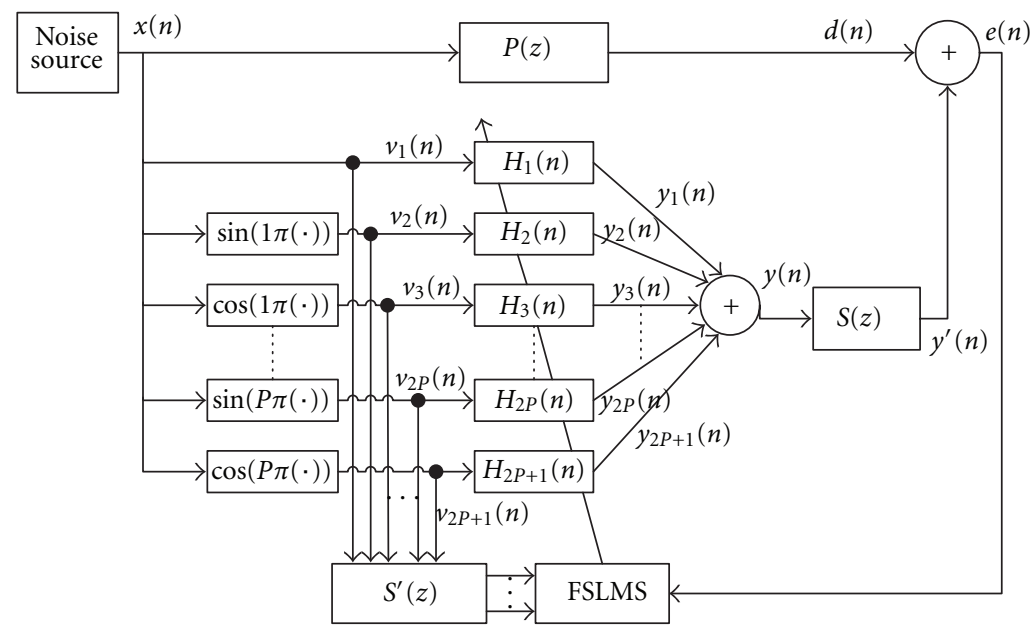

FIGURE 4: FSXLMS for the feedforward ANC system.

for hardware implementation [34]. The general functional expansion equation that expands an input signal is given by

$$
y(n)=\sum_{i=1}^{K} H_{i}(n) \Psi_{i}\{\mathbf{x}(n)\},
$$

where $\mathbf{x}(n)$ and $y(n)$ represent the input vector and the output of the nonlinear system, respectively. $H_{i}(n)$ is the $i$ th coefficient filter at time $(n), \Psi$ is an orthogonal basis functional expansion. Several function expansions in the form of approximate and exact trigonometric, Legendre (L-FLANN), Chebyshev (C-FLANN), Hammerstein (HFLANN), and Piecewise linear expansion (PWL) have been implemented [14-16].

The block diagram of the FSLMS based on FLNN is shown in Figure 4.

From Figure 4, the expansion function $\Psi$ used is the exact trigonometric functions of order $P$, and

$$
y(n)=\sum_{i=1}^{2 P+1} y_{i}(n),
$$

and $y_{i}(n)$ can be further defined as

$$
y_{i}(n)=v_{i}(n) * H_{i}(n)
$$

where $v_{i}(n)$ and $H_{i}(n)$ represent the $i$ th expanded input signal and the $i$ th filter coefficients, respectively, and they are expressed as

$$
\begin{aligned}
& v_{1}(n)=x(n), \\
& v_{2}(n)=\sin \{\pi x(n)\}, \\
& v_{3}(n)=\cos \{\pi x(n)\}, \\
& \vdots \\
& v_{2 P}(n)=\sin \{P \pi x(n)\}, \\
& v_{2 P+1}(n)=\cos \{P \pi x(n)\} ; \\
& H_{i}(n)=\left[h_{i, 1}(n), h_{i, 2}(n), \ldots, h_{i, M}(n)\right] .
\end{aligned}
$$

The weight updates equations can be derived using the steepest descent algorithm to minimise the squared error function $e^{2}(n)$ and can be expressed as [17]

$$
H_{i}(n+1)=H_{i}(n)+\mu e(n)\left\{s(n) * v_{i}(n)\right\} .
$$

The disadvantage of the FSLMS algorithm is the extra computation required by the functional expansion block. The choice of the expansion function depends on the strength of nonlinearity and sets the computational complexity of the model. Thus, numerous expansion functions have been used to achieve compromised design between computational complexity and performance [14]. However, under higher degree of nonlinearities, the FSLMS performs better compared to VFXLMS and BFXLMS.

In NANC system, appropriate nonlinear algorithm is required in compensating the nonlinearity effects. Improved noise reduction can sometimes be achieved at the expense of high computation load. It is important to understand the trade off between performance and computational load in the design process. In the next section, a performance comparison between the nonlinear algorithms is investigated by means of simulation. In achieving fair comparison, the nonlinear algorithms are applied under the same system conditions and nonlinearity sources (type of reference noise, primary and secondary path transfer functions).

\section{Computer Simulation and Performance Comparison}

The performances of the three nonlinear algorithms with the associated models are compared. The normalized mean square error (NMSE) after convergence versus the number of iterations is used as the performance criteria [16] and is defined as

$$
\mathrm{NMSE}=10 \log _{10}\left(\frac{E\left\{e^{2}(n)\right\}}{\sigma_{d}^{2}}\right),
$$

where $\sigma_{d}^{2}$ is the variance of the primary noise at the cancellation point, and $E\left\{e^{2}(n)\right\}$ is the expected value of 
TABLE 1: NMSE simulation results summary.

\begin{tabular}{|c|c|c|c|c|c|c|}
\hline \multirow{2}{*}{$\begin{array}{l}\text { NANC } \\
\text { algorithm }\end{array}$} & \multicolumn{2}{|c|}{ Chaotic reference noise } & \multicolumn{2}{|c|}{ Nonlinear primary path } & \multicolumn{2}{|c|}{ Loudspeaker saturation $85 \%$} \\
\hline & $\operatorname{NMSE}(\mathrm{dB})$ & Step size & NMSE (dB) & Step size & NMSE $(\mathrm{dB})$ & Step size \\
\hline FXLMS & -39 & $\mu=2 \times 10^{-2}$ & -22 & $\mu=2 \times 10^{-3}$ & -27 & $\mu=2 \times 10^{-3}$ \\
\hline \multirow{2}{*}{ VFXLMS } & & $\mu_{1}=2 \times 10^{-2}$ & & $\mu_{1}=2 \times 10^{-3}$ & & $\mu_{1}=2 \times 10^{-3}$ \\
\hline & -39 & $\mu_{2}=2 \times 10^{-5}$ & -24 & $\mu_{2}=2 \times 10^{-5}$ & -26 & $\mu_{2}=2 \times 10^{-5}$ \\
\hline \multirow{3}{*}{ BFXLMS } & & $\mu_{\mathrm{A}}=2 \times 10^{-2}$ & & $\mu_{\mathrm{A}}=2 \times 10^{-3}$ & & $\mu_{\mathrm{A}}=2 \times 10^{-3}$ \\
\hline & -48 & $\mu_{\mathrm{B}}=2 \times 10^{-4}$ & -22 & $\mu_{\mathrm{B}}=2 \times 10^{-3}$ & -29 & $\mu_{\mathrm{B}}=2 \times 10^{-3}$ \\
\hline & & $\mu_{\mathrm{C}}=2 \times 10^{-4}$ & & $\mu_{\mathrm{C}}=2 \times 10^{-4}$ & & $\mu_{\mathrm{C}}=2 \times 10^{-6}$ \\
\hline FSLMS & -31 & $\mu=2 \times 10^{-3}$ & -18 & $\mu=2 \times 10^{-3}$ & -29 & $\mu=2 \times 10^{-3}$ \\
\hline
\end{tabular}

the squared error. The expected value is approximated by averaging the recorded squared error of fifty independent trials (simulations). In the simulation, the primary noise signal $d(n)$ is generated using the third-order polynomial model defined by (3), (4), and (5). The secondary path impulse response $s(n)$ and its estimate $s^{\prime}(n)$ used in the simulation are linear minimum phase [15] defined by

$$
s(n)=s^{\prime}(n)=\delta(n-2)+0.5 \delta(n-3) .
$$

The saturation characteristic of the loudspeaker is modelled by saturating the controller output to a certain level. This level of saturation is obtained by setting a clipping threshold to the controller output signal at $85 \%$ of the maximum signal value.

Two reference noises are used in this simulation to investigate the performances of the algorithms. The first noise process is represented by a multiharmonic signal $[6$, 17]. The multiharmonic sum of three sine waves of $500 \mathrm{~Hz}$, $400 \mathrm{~Hz}$, and $300 \mathrm{~Hz}$ sampled at the rate of 8000 samples/sec was used and is given by

$$
\begin{aligned}
x(n)= & \sin \left(\frac{2 \pi 500 n}{8000}\right)+\sin \left(\frac{2 \pi 400 n}{8000}\right) \\
& +\sin \left(\frac{2 \pi 300 n}{8000}\right)+\xi(n),
\end{aligned}
$$

where $\xi(n)$ is a white noise process with Gaussian distribution. The signal to noise power ratio (SNR) was set at $40 \mathrm{~dB}$. The second noise process is logistic chaotic noise. The logistic chaotic noise is a second-order white and predictable nonlinear process. It was generated using (2). This nonlinear noise process was then normalised to have unity signal power to prevent gradient noise amplification in the weights update equation.

Three experiments were simulated to compare the performance of the NANC algorithms with the FXLMS algorithm. In each experiment, only a single nonlinearity source is introduced in the ANC system at any time. This was done to evaluate the performance of the NANC and the FXLMS algorithms in the presence of the corresponding nonlinearity source individually. In all experiments, the memory tap of the Volterra filter $(M)$, secondary path filter length $(L)$ and the functional expansion order of the FLNN filter $(P)$ were set to 10,4 , and 3 , respectively. Figures 5, 6, and 7

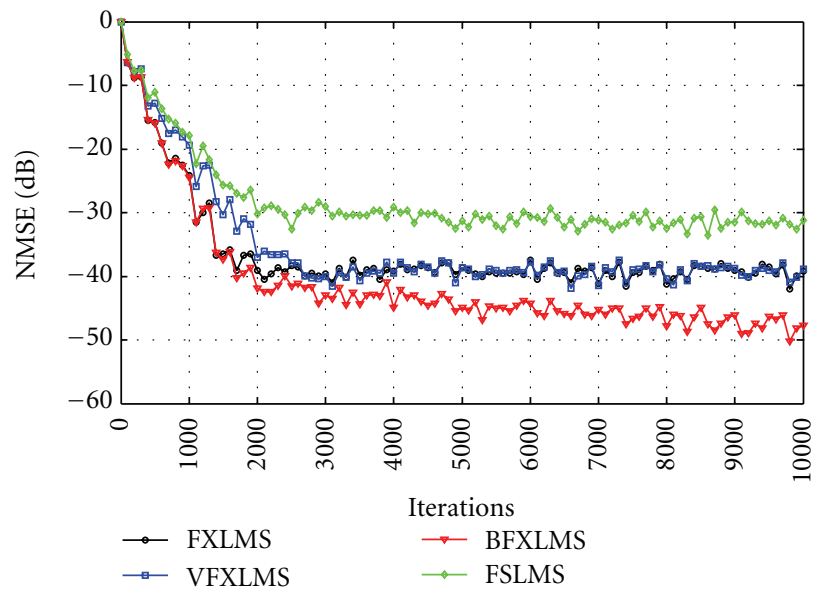

FIgURE 5: NANC and FXLMS algorithms performance in the presence of nonlinear chaotic noise.

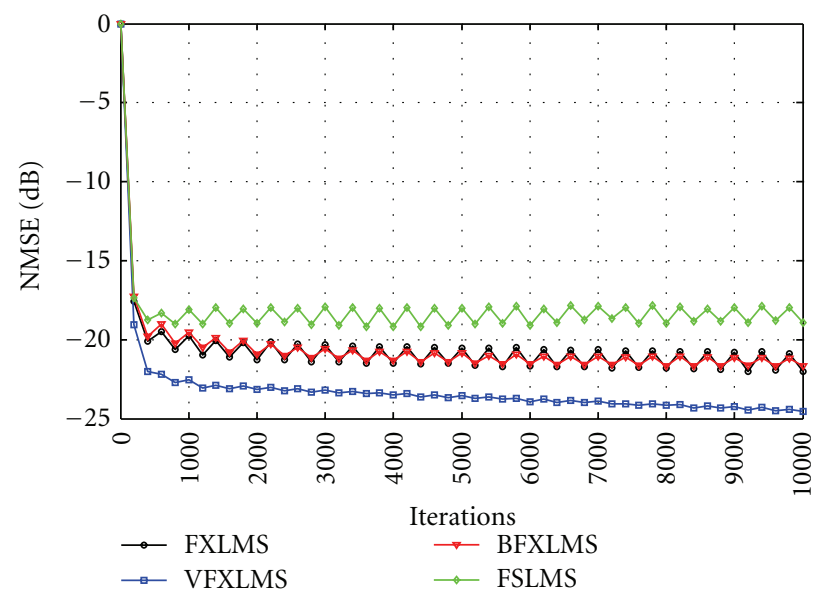

Figure 6: NANC and FXLMS algorithms performance in the presence of nonlinear primary path.

depict the noise reduction performance in the presence of chaotic noise, nonlinear primary path, and saturation effects of secondary path, respectively. Table 1 presents the summary of the simulation results obtained with the three nonlinearity sources for different NANC algorithms after convergence at the $10^{4}$ iteration. 
TABLE 2: Complexity comparison of NANC algorithms.

\begin{tabular}{|c|c|c|c|c|c|c|c|}
\hline NANC algorithm & Op. & $\begin{array}{c}\text { Controller } \\
\text { output (stage 1) }\end{array}$ & $\begin{array}{l}\text { Filtered } \mathrm{x} \\
\text { (stage 2) }\end{array}$ & $\begin{array}{l}\text { Weights update } \\
\text { (stage } 3 \text { ) }\end{array}$ & $\begin{array}{l}\sin (\cdot) \& \\
\cos (\cdot)\end{array}$ & Total & $\begin{aligned} M & =10 \\
L & =4\end{aligned}$ \\
\hline \multirow{2}{*}{ FXLMS } & $\times$ & $M$ & $L$ & $M+1$ & - & $2 M+L+1$ & 25 \\
\hline & + & $M-1$ & $L-1$ & $M$ & - & $2 M+L-2$ & 22 \\
\hline \multirow{2}{*}{$\begin{array}{l}\text { VFXLMS } \\
2^{\mathrm{Md}} \text { order }\end{array}$} & $\times$ & $M(M+5) / 2$ & $L$ & $M^{2}+2 M+1$ & - & $\left(3 M^{2}+9 M+2 L+2\right) / 2$ & 200 \\
\hline & + & $\left(M^{2}+3 M-4\right) / 2$ & $L-1$ & $M(M+1) / 2$ & - & $M 2+2 M+L-3$ & 121 \\
\hline \multirow{2}{*}{ BFXLMS } & $\times$ & $2 M^{2}+4 M+1$ & $2 L$ & $M^{2}+4 M+4$ & - & $3 M^{2}+8 M+2 L+5$ & 393 \\
\hline & + & $M^{2}+3 M-2$ & $2 L-2$ & $M^{2}+3 M+1$ & - & $2 M 2+6 M+2 L-3$ & 265 \\
\hline \multirow{2}{*}{$\begin{array}{l}\text { FSLMS } \\
P=3\end{array}$} & $\times$ & $7 M$ & $7 L M$ & $14 M$ & $6 M$ & $7 M(L+3)$ & 490 \\
\hline & + & $7 M-1$ & $7 M-L+1$ & $7 M-1$ & - & $21 M-L-1$ & 205 \\
\hline
\end{tabular}

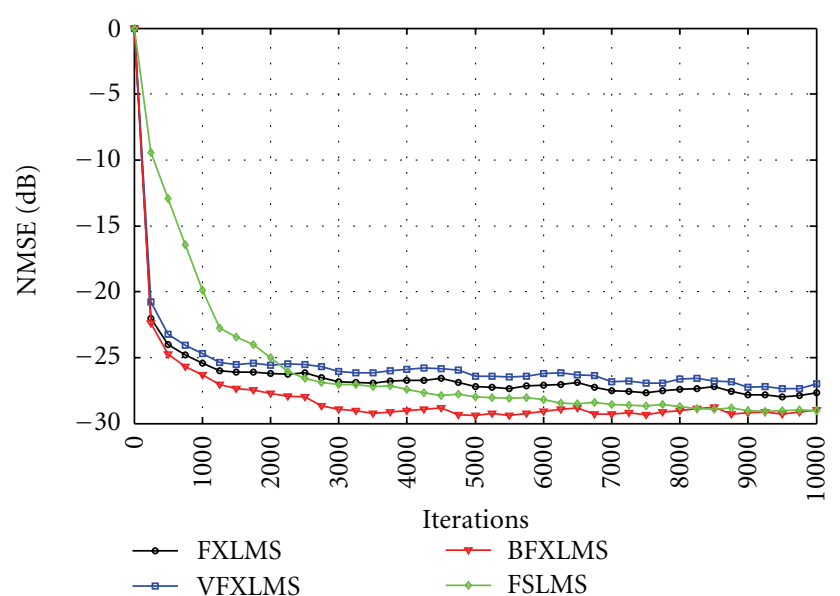

FIGURE 7: NANC and FXLMS algorithms performance in the presence of loudspeaker saturation.

Figure 5 shows the NMSE of different NANC algorithms with the presence of chaotic noise. The simulation shows that the BFXLMS is the most effective algorithm. However, the FXLMS algorithm can still achieve a noise reduction of $81.25 \%$ of the reduced noise achieved by the BFXLMS algorithm. Figure 6 shows the NMSE of different NANC algorithms in the presence of nonlinear primary path given by (3), (4), and (5). Although the second-order VFXLMS algorithm gives the most effective noise reduction, the FXLMS is still capable of achieving $91.66 \%$ of the VFXLMS performance. Figure 7 shows the NMSE for different NANC algorithms in the presence of saturation. Here, it is evident that FSLMS and BFXLMS perform equally yet better than VFXLMS and FXLMS. However, the FXLMS algorithm can still achieve a noise reduction of $93.10 \%$ of the reduced noise achieved by both the FSLMS and the BFXLMS algorithms.

\section{Computational Complexity}

Computational complexity is an important issue when implementing the algorithm in real-time application. In feed forward ANC structure, three stages of signal processing computation are performed. These stages of computation are

(1) generating the control signal,

(2) filtering the reference signal through the estimated secondary path,

(3) updating the controller weights.

Table 2 summarizes the computational complexities of the algorithms for each processing stage. From the table, the FXLMS has the least number of multiplications and additions followed by the VFXLMS. Taking into account only the number of multiplications which dominates the computational requirements, the FXLMS computational load is only $12.5 \%$ in comparison to that of the VFXLMS.

\section{Conclusion}

In this paper, the performance and computational load of three nonlinear active noise control algorithms and the conventional FXLMS algorithm are compared. The nonlinearity sources that affect the system are categorised into noise process, primary and secondary acoustic paths, and the actuators which consist of the loudspeaker, microphone, and amplifier. Three NANC algorithms, namely, VFXLMS, BFSLMS, and FSLMS were simulated on a feedforward ANC system. The results from this study suggest that, in the presence of nonlinearity sources, the FXLMS algorithm still gives an acceptable performance compared to nonlinear control algorithms at the fraction of computational load.

\section{References}

[1] F. Russo and G. L. Sicuranza, "Accuracy and performance evaluation in the genetic optimization of nonlinear systems for active noise control," IEEE Transactions on Instrumentation and Measurement, vol. 56, no. 4, pp. 1443-1450, 2007.

[2] J. Poxon, P. Jennings, and M. Allman-Ward, "Development of a hybrid electric vehicle (HEV) model for interactive customer assessment of sound quality," in Proceedings of the Hybrid and Eco Friendly Vehicles Conference 2008 (HEVC '08), pp. 1-4, December 2008. 
[3] G. P. van den Berg, "Effects of the wind profile at night on wind turbine sound," Journal of Sound and Vibration, vol. 277, no. 4-5, pp. 955-970, 2004.

[4] C. Hansen, Noise Control: From Concept to Application, Taylor \& Francis, London, UK, 2005.

[5] C. Y. Chang and F. B. Luoh, "Enhancement of active noise control using neural-based filtered-X algorithm," Journal of Sound and Vibration, vol. 305, no. 1-2, pp. 348-356, 2007.

[6] R. Napoli and L. Piroddi, "Nonlinear active noise control using narx model structure selection," in Proceedings of the American Control Conference (ACC '09), pp. 5616-5621, June 2009.

[7] E. P. Reddy, D. P. Das, and K. M. M. Prabhu, "Fast exact multichannel FSLMS algorithm for active noise control," Signal Processing, vol. 89, no. 5, pp. 952-956, 2009.

[8] T. Li and J. Jiang, "Adaptive second-order volterra filteredX RLS algorithms with sequential and partial updates for nonlinear active noise control," in Proceedings of the Industrial Electronics and Applications (ICIEA '09), pp. 1625-1630, May 2009.

[9] T. Li and J. Jiang, "Adaptive Volterra filters for active control of nonlinear noise processes," IEEE Transactions on Signal Processing, vol. 49, no. 8, pp. 1667-1676, 2001.

[10] D. Zhou and V. DeBrunner, "Efficient adaptive nonlinear filters for nonlinear active noise control," IEEE Transactions on Circuits and Systems I: Regular Papers, vol. 54, no. 3, pp. 669$681,2007$.

[11] Y. Zhou, Q. Zhang, X. Li, and W. Gan, "Model-free control of nonlinear noise processes based on c-flan," Advances in Soft Computing, vol. 56, pp. 535-541, 2009.

[12] Z. Haiquan and Z. Jiashu, "Filtered-s Lyapunov algorithm for active control of nonlinear noise processes," in Proceedings of the 9th International Conference on Signal Processing (ICSP '08), pp. 311-314, October 2008.

[13] H. Zhao, X. Zeng, and J. Zhang, "Adaptive reduced feedback FLNN filter for active control of nonlinear noise processes," Signal Processing, vol. 90, no. 3, pp. 834-847, 2010.

[14] G. L. Sicuranza and A. Carini, "On the accuracy of generalized hammerstein models for nonlinear active noise control," in Proceedings of the IEEE Instrumentation and Measurement Technology Conference (IMTC '06), pp. 1411-1416, Sorrento,Italy, April 2006.

[15] G. L. Sicuranza and A. Carini, "Piecewise-linear expansions for nonlinear active noise control," in Proceedings of the IEEE International Conference on Acoustics, Speech and Signal Processing (ICASSP '06), pp. III209-III212, Toulouse, France, May 2006.

[16] D. P. Das and G. Panda, "Active mitigation of nonlinear noise processes using a novel filtered-s LMS algorithm," IEEE Transactions on Speech and Audio Processing, vol. 12, no. 3, pp. 313-322, 2004.

[17] S. M. Kuo and H. T. Wu, "Nonlinear adaptive bilinear filters for active noise control systems," IEEE Transactions on Circuits and Systems I: Regular Papers, vol. 52, no. 3, pp. 617-624, 2005.

[18] Q. Z. Zhang, W. S. Gan, and Y. L. Zhou, "Adaptive recurrent fuzzy neural networks for active noise control," Journal of Sound and Vibration, vol. 296, no. 4-5, pp. 935-948, 2006.

[19] Q. Z. Zhang and W. S. Gan, "Active noise control using a simplified fuzzy neural network," Journal of Sound and Vibration, vol. 272, no. 1-2, pp. 437-449, 2004.

[20] S. M. Kuo, H. T. Wu, F. K. Chen, and M. R. Gunnala, "Saturation effects in active noise control systems," IEEE Transactions on Circuits and Systems I: Regular Papers, vol. 51, no. 6, pp. 1163-1171, 2004.
[21] M. Soria-Rodríguez, M. Gabbouj, N. Zacharov, M. S. Hämäläinen, and K. Koivuniemi, "Modeling and real-time auralization of electrodynamic loudspeaker non-linearities," in Proceedings of the IEEE International Conference on Acoustics, Speech, and Signal Processing, pp. iv-81-iv-84, Montreal, Canada, May 2004.

[22] A. N. Birkett and R. A. Goubran, "Limitations of handsfree acoustic echo cancellers due to nonlinear loudspeaker distortion and enclosure vibration effects," in Proceedings of the 1995 IEEE ASSP Workshop on Applications of Signal Processing to Audio and Acoustics, pp. 103-106, October 1995.

[23] D. Sankar and T. Thomas, "Nonlinear modeling of loudspeaker using adaptive second order Volterra filters," in Proceedings of the International Conference on Information and Communication Technology in Electrical Sciences (ICTES '07), pp. 87-92, Tamilnadu, India, December 2007.

[24] D. Hongyun and Z. Wei-Ping, "Compensation of loudspeaker nonlinearity in acoustic echo cancellation using raised-cosine function," IEEE Transactions on Circuits and Systems II: Express Briefs, vol. 53, no. 11, pp. 1190-1194, 2006.

[25] L. Zhenyan, D. Huachun, and Q. Taifan, "Apply genetic algorithm to parameter estimation in chaotic noise," in Proceedings of the 6th International Conference on Signal Processing, vol. 2, pp. 1399-1402, August 2002.

[26] H. Leung, "Applying chaos to radar detection in an ocean environment: an experimental study," IEEE Journal of Oceanic Engineering, vol. 20, no. 1, pp. 56-64, 1995.

[27] S. H. Kellert, In the Wake of Chaos: Unpredictable Order in Dynamical Systems, University of Chicago Press, Chicago, Ill, USA, 1993.

[28] T. Matsuura, T. Hiei, H. Itoh, and K. Torikoshi, "Active noise control by using prediction of time series data with a neural network," in Proceedings of the 1995 IEEE International Conference on Systems, Man and Cybernetics, Intelligent Systems for the 21st Century, vol. 3, pp. 2070-2075, October 1995.

[29] P. Strauch and B. Mulgrew, "Active control of nonlinear noise processes in a linear duct," IEEE Transactions on Signal Processing, vol. 46, no. 9, pp. 2404-2412, 1998.

[30] K. Wolfgang, Active Attenuation of Nonlinear Sound, Thousand Oaks, Calif, USA, 1999.

[31] S. M. Kuo and D. R. Morgan, "Active noise control: a tutorial review," Proceedings of the IEEE, vol. 87, no. 6, pp. 943-973, 1999.

[32] S. J. Elliott, Signal Processing for Active Control. Signal Processing and Its Applications, Academic Press, San Diego, Calif, USA, 2001.

[33] L. A. Aguirre and S. A. Billings, "Dynamical effects of overparametrization in nonlinear models," Physica D, vol. 80, no. 1-2, pp. 26-40, 1995.

[34] Y. H. Pao and Y. Takefuji, "Functional-link net computing: theory, system architecture, and functionalities," Computer, vol. 25, no. 5, pp. 76-79, 1992. 

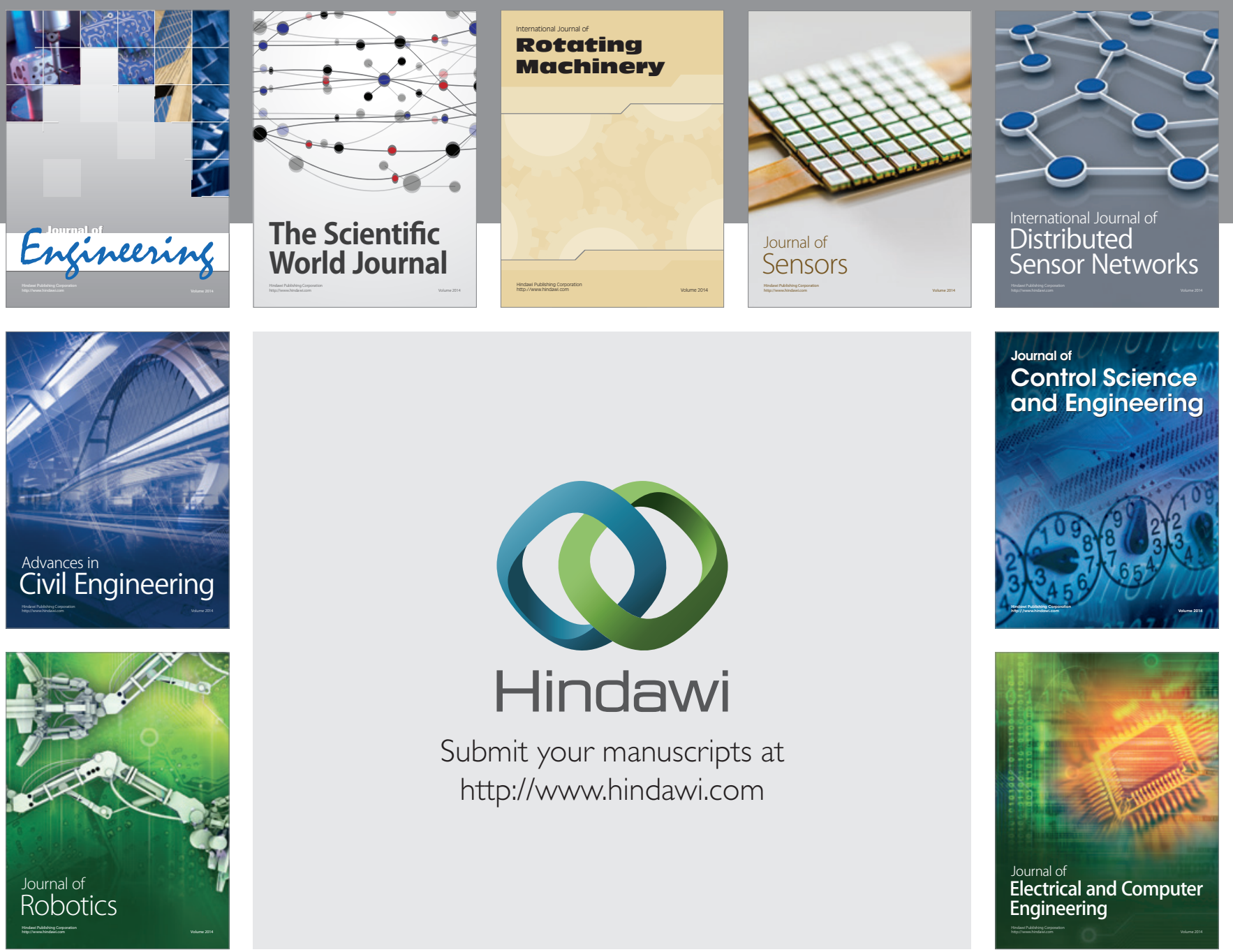

Submit your manuscripts at

http://www.hindawi.com
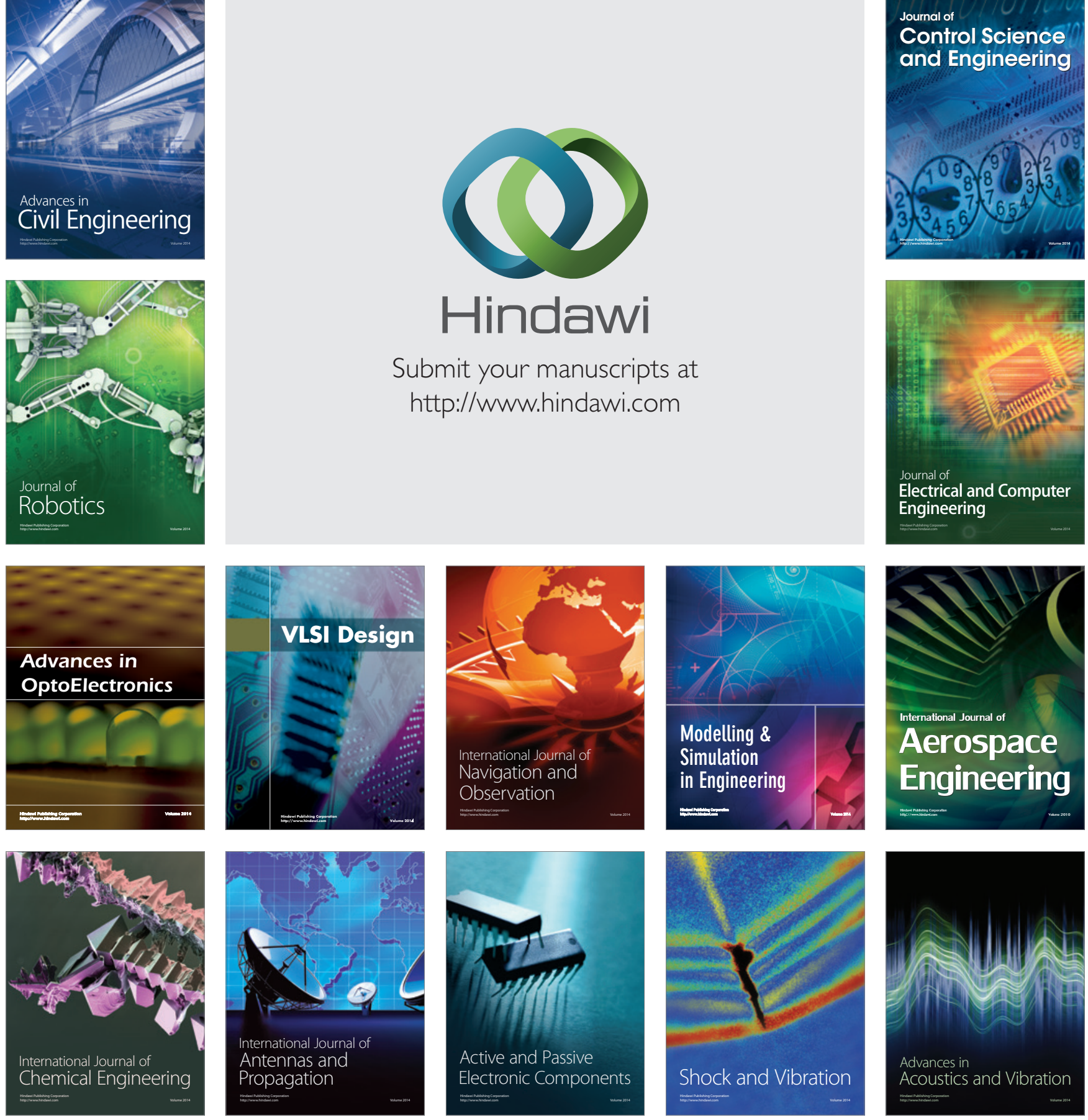